\title{
Delirium and geriatric syndromes in hospitalized older patients: Results from World Delirium Awareness Day
}

\author{
Gökcen UMURCA ${ }^{1}$ (D), Busra CAN² (D), Birkan ILHAN³ (D), Asli TUFAN² (D) \\ ${ }^{1}$ Department of Internal Medicine, School of Medicine, Marmara University, Istanbul, Turkey. \\ ${ }^{2}$ Division of Geriatrics, Department of Internal Medicine, School of Medicine, Marmara University, Istanbul, Turkey. \\ ${ }^{3}$ Division of Geriatrics, Department of Internal Medicine, Sisli Hamidiye Etfal Teaching and Research Hospital, Istanbul, Turkey.
}

Corresponding Author: Busra CAN

E-mail: alpabusra@hotmail.com

Submitted: 23.08.2021 Accepted: 17.11.2022

\begin{abstract}
Objective: To determine the point prevalence of delirium and the associated risk factors in geriatric inpatients.

Patients and Methods: Sixty-two hospitalized patients aged 60 years and over were recruited. The Mini Nutritional Assessment-Short Form (MNA-SF), the FRAIL scale, Katz Activities of Daily Living (ADL), and Lawton-Brody Instrumental ADL (IADL) questionnaires were administered. A delirium evaluation test (Confusion Assessment Method-CAM) was performed for diagnosing delirium. Mortality was evaluated one month after the index date.

Results: The median age was 71.5 (range, 60-96) years. Delirium was detected in $29 \%$ of the patients. Frailty and dementia were associated with delirium $(\mathrm{p}<0.001$ and $\mathrm{p}=0.001$; respectively). Polypharmacy, indwelling urinary catheter, and low albumin were also related to delirium $(\mathrm{p}=0.025, \mathrm{p}=0.007$, and $\mathrm{p}=0.002$; respectively). Age over 70 years, low Katz ADL and low MNA-SF scores were found to be independently associated with delirium in multivariate regression analysis models. The median hospitalization time was longer in the delirium group ( $\mathrm{p}=0.029)$. Survival analysis at one month showed no significant difference between the delirium and non-delirium groups.

Conclusion: Age over 70 years, impaired functionality in ADL and malnutrition were independently associated with delirium.

Keywords: Delirium, Functionality, Geriatrics, Geriatric syndromes, Malnutrition
\end{abstract}

\section{INTRODUCTION}

Delirium is a neuropsychiatric disorder characterized by disturbances in attention, orientation and cognition, often triggered by stressors such as medical causes, pain and/or medication [1]. Although, delirium can be seen at all ages, children and older adults have the highest risk. The prevalence of delirium varies between $9 \%$ and $89 \%$, depending on the sample and the hospital department; the incidence ranges from $18 \%$ to $35 \%$ in internal medicine and geriatrics clinics [2]. Delirium is associated with increased mortality, prolonged hospital stay, progression of dementia, impaired quality of life, and increased health care costs [3-6]. Delirium screening has been accepted as an important quality indicator for geriatric care in many countries around the world and delirium prevention interventions have become an important part of geriatric patient care programs $[7,8]$.

Delirium is a partially preventable syndrome. Unfortunately, due to its fluctuating course and diverse symptoms, its diagnosis is often delayed or missed [9]. Early recognition of patients at risk of delirium development may enable preventive action, thereby reducing the associated mortality. The aim of this study was to determine delirium prevalence, associated risk factors, and mortality rates in hospitalized older adults. Our secondary objective was to raise awareness about delirium with the point prevalence study we conducted on March 13th, World Delirium Awareness Day.

\section{PATIENTS and METHODS}

\section{Study Design and Participants}

The research was conducted on the index date of March 13th, World Delirium Awareness Day, to determine the point prevalence of delirium in the internal medicine clinic of a university hospital. The study sample consisted of patients aged 60 years and over, 
who were hospitalized in an internal medicine clinic for more than 24 hours. Exclusion criteria were coma, end of life status, and refusal of consent. Sixty-two (26 women, 36 men) eligible patients were recruited. All data were collected from the patients themselves or their first-degree relatives, the hospital electronic data system, and patient records. Written informed consent was obtained from all participants. For patients who were unable to give informed consent, written informed consent was provided by a proxy on behalf of the patient. The trial protocol was approved by Clinical Research Ethics Committee of Marmara University, School of Medicine (Protocol code: 09.2019.273).

Demographic information including age, sex, height, weight and comorbidities were recorded. Medication history was obtained from patients' medical records. The Mini Nutritional Assessment Short Form (MNA-SF) [10, 11], Katz Activities of Daily Living (ADL) and Lawton-Brody Instrumental Activities of Daily Living (IADL) $[12,13]$ were administered to evaluate the patients' nutritional and functional status. The MNA-SF is a screening tool scored out of 14 points. An MNA-SF score of 0-7 indicates malnutrition, and a score of 8-11 indicates malnutrition risk. Functionality was evaluated using the sixitem Katz ADL scores and eight - item Lawton-Brody IADL scores. The scores for each item were determined as 1,2 , and 3 if the patient was totally dependent, partially dependent, and independent in carrying out the related activity, respectively. For the ADL, 6, 7-12 and 13-18 points, and for the IADL, 8, 9-16 and 17-24 points correspond to dependency, partial dependency, and independency, respectively.

Possible risk factors for delirium were evaluated including hearing impairment (hearing aid use), visual impairment (use of glasses), polypharmacy (five or more medications) [14], number of medications prescribed, presence of a urinary catheter, presence of a peripheral-central vein catheter, presence of nasogastric (NG)/nasoduodenal (ND) feeding tubes/ percutaneous endoscopic gastrostomy (PEG), and physical restraints. To evaluate hyponatremia, kidney damage, anemia, and inflammation, serum sodium, creatinine, hemoglobin, and C-reactive protein (CRP) values were recorded, respectively.

Frailty was assessed using the FRAIL scale, which consists of five questions on Fatigue, Resistance, Ambulation, Illness, and Loss of Weight. The FRAIL scale scores range from 0 to 5, where 3 to 5 represent frailty and 1 to 2 represent pre-frailty [15].

Patients were screened for delirium using the Confusion Assessment Method (CAM) on the index date [16]. CAM consists of four features: 1) mental disorder of acute onset or fluctuating course, 2) disturbance of attention, 3) disorganized thinking, and 4) altered consciousness. To diagnose delirium, features 1, 2 and either 3 or 4 must be present.

Mortality data were obtained from the "Ministry of HealthDeath Reporting System" one month after the index date.

\section{Statistical Analysis}

The demographic and baseline characteristics of the subjects were summarized using mean and standard deviation (SD0 (or median and minimum-maximum values for non - normally distributed variables) if the variables were continuous, or frequencies and percentages if the variables were categorical. Subjects with and without delirium were compared using Student's t-test for continuous variables with normal distribution or the MannWhitney U test for those without. The consistency of the continuous data to normal distribution was evaluated using the KolmogorovSmirnov/Shapiro-Wilk tests. The Chi-square test or Fischer's exact test was used to compare categorical variables. Multivariate logistic regression analysis was used to evaluate the relationship between delirium and variables that reached statistical significance in univariate logistic regression analysis such as Katz ADL, MNA-SF, the presence of a urinary catheter, advanced age, and the number of drugs used. Multicollinearity was checked among parameters using Pearson's, Spearman's or Kendall's tau-b correlation analyses. Finally, survival analysis was performed using the Kaplan Meier test one month after the index date. All tests were two-sided and statistical significance was set at $\mathrm{p}=0.05$. All statistical tests were performed using the IBM Statistical Package for the Social Sciences (SPSS) Version 22.0 software package.

\section{RESULTS}

A total of 62 patients were included in the study, $36(58.1 \%)$ were male and $26(41.9 \%)$ were female (Table I). The median age of patients was 71.5 (range, 60-96) years.

Table I. Sociodemographic characteristics $(n=62)$

\begin{tabular}{|c|c|}
\hline Variables & Values \\
\hline \multicolumn{2}{|l|}{ Sex, n (\%) } \\
\hline Female & $26(41.9)$ \\
\hline Male & $36(58.1)$ \\
\hline Age, median (min-max) & $71.5(60-96)$ \\
\hline \multicolumn{2}{|l|}{ Marital status, n (\%) } \\
\hline Married & $36(58.1)$ \\
\hline Widow/Divorced/Separated & $24(38.7)$ \\
\hline Single & $2(3.2)$ \\
\hline \multicolumn{2}{|l|}{ Education, $\mathbf{n}(\%)$} \\
\hline Illiterate & $20(32.3)$ \\
\hline Primary school & $30(48.4)$ \\
\hline Secondary school & $3(4.8)$ \\
\hline High school & $5(8.1)$ \\
\hline University & $4(6.5)$ \\
\hline \multicolumn{2}{|l|}{ Residency status, $\mathbf{n}(\%)$} \\
\hline Home with family members & $58(93.5)$ \\
\hline Nursing homes & $4(6.5)$ \\
\hline \multicolumn{2}{|l|}{ Smoking, $\mathbf{n}(\%)$} \\
\hline Active smoker & $2(3.2)$ \\
\hline Quit smoking & $32(51.6)$ \\
\hline Nonsmoker & $28(45.2)$ \\
\hline
\end{tabular}

The medical characteristics, comorbidities, diagnoses, and laboratory findings of the patients on the index day are shown in Supplementary Tables I and II. 
Table II. Relationship between delirium and frailty

\begin{tabular}{|llll|} 
& $\begin{array}{l}\text { Delirium } \\
\mathbf{n = 1 8 ( 2 9 \% )}\end{array}$ & $\begin{array}{l}\text { No delirium } \\
\mathbf{n = 4 4}(\mathbf{7 1 \% )})\end{array}$ & p value \\
\hline Frailty, $\mathbf{n}(\%)$ & & $0.000^{\wedge}$ \\
\hline Pre-frail (FRAIL score 1-2) & $0(0)$ & $21(47.7)$ & \\
\hline Frail (FRAIL score $>3)$ & $18(100)$ & $23(52.3)$ & \\
\hline
\end{tabular}

$\wedge$ Fischer's exact test

Delirium was detected in $29 \%(n=18)$ of the patients using CAM. $72 \%(n=13)$ of the patients with delirium had the hypoactive subtype. Patients with delirium were significantly older than those without delirium (76 (range, 61-96) years vs. 69 (range, 60-90) years; $\mathrm{p}=0.020$ ). Serum albumin levels were significantly lower in patients with delirium (2.5 (range, 1.83.3) $\mathrm{g} / \mathrm{L}$ vs 3.1 (range, 2.4-4.4) $\mathrm{g} / \mathrm{L} ; \mathrm{p}=0.002$ ). Delirium was detected in all five $(27.8 \%)$ patients with dementia. Dementia was found to be the only comorbidity associated with delirium $(p=0.001)$. Hypertension, diabetes mellitus and malignancies were the three most common comorbid conditions (Supplementary Table I).

Table III. Relationship between delirium and other geriatric syndromes

\begin{tabular}{|c|c|c|c|}
\hline & $\begin{array}{c}\text { Delirium } \\
\mathrm{n}=18(29 \%)\end{array}$ & $\begin{array}{l}\text { No delirium } \\
\mathrm{n}=44(71 \%)\end{array}$ & $p$ value \\
\hline MNA-SF score, mean (SD) & $4.6( \pm 2.4)$ & $8.2( \pm 2.8)$ & $0.000^{\&}$ \\
\hline Nutritional status, $\mathbf{n}(\%)$ & & & $0.004^{\#}$ \\
\hline $\begin{array}{l}\text { Normal nutritional status (MNA-SF } \\
\text { score 12-14) }\end{array}$ & $0(0)$ & $7(15.9)$ & \\
\hline $\begin{array}{l}\text { At risk of malnutrition (MNA-SF } \\
\text { score 8-11) }\end{array}$ & $2(11.1)$ & $18(40.9)$ & \\
\hline Malnourished (MNA-SF score 0-7) & $16(88.9)$ & $19(43.2)$ & \\
\hline Katz ADL score, median (min-max) & $7(6-18)$ & $17(8-18)$ & $0.000^{+}$ \\
\hline $\begin{array}{l}\text { Functional status according to Katz } \\
\text { score, } \mathbf{n}(\%)\end{array}$ & & & $0.000^{*}$ \\
\hline Severe dependency $(0-6)$ & $9(50)$ & $0(0)$ & \\
\hline Mild dependency (7-12) & $6(33.3)$ & $14(31.8)$ & \\
\hline Independency (13-18) & $3(16.7)$ & $30(68.2)$ & \\
\hline $\begin{array}{l}\text { Lawton IADL score, median (min- } \\
\max )\end{array}$ & $9(8-19)$ & $16(8-24)$ & $0.000^{+}$ \\
\hline $\begin{array}{l}\text { Functional status according to } \\
\text { Lawton score, } \mathrm{n}(\%)\end{array}$ & & & $0.000^{\#}$ \\
\hline Severe dependency $(0-8)$ & $8(44.4)$ & $1(2.3)$ & \\
\hline Mild dependency (9-16) & $9(50)$ & $22(50)$ & \\
\hline Independency (17-24) & $1(5.6)$ & $21(47.7)$ & \\
\hline \multicolumn{4}{|c|}{$\begin{array}{l}\text { MNA-SF: Mini nutritional assessment short form, ADL: Activities of daily } \\
\text { living, }\end{array}$} \\
\hline $\begin{array}{l}\text { IADL: Instrumental activities of dai } \\
\text { Student t test, + Mann Whitney U test }\end{array}$ & ly living, SD: & Standard & iation \\
\hline
\end{tabular}

Delirium was associated with old age, defined as age over 70 years $(p=0.030)$. Patients with delirium were treated with a higher number of drugs (11 vs 9; $\mathrm{p}=0.025)$. Antipsychotics $(\mathrm{p}=0.006)$ and nutritional support products $(\mathrm{p}<0.001)$ were more commonly used among patients with delirium. There was no significant difference in terms of other drugs ( $\mathrm{p}>0.05)$. Indwelling urinary catheter and delirium were also significantly associated $(\mathrm{p}=0.007)$.

Our study sample consisted only of pre-frail and frail patients. All patients with delirium were frail $(n=18,100 \%)$ and frailty was strongly associated with delirium $(\mathrm{p}<0.001)$ (Table II). Patients with delirium had significantly lower MNA-SF (4.6 vs. 8.2; $\mathrm{p}<0.001$ ), Katz ADL (7 vs. 17; $<<0.001$ ) and Lawton IADL (9 vs. 16; $\mathrm{p}<0.001)$ scores (Table III).

Multivariate regression analysis was performed to determine independent risk factors for delirium. Variables with statistical significance in univariate analysis or univariate logistic regression analysis (Table IV) such as age over 70 years, presence of an indwelling urinary catheter, the number of drugs used, serum albumin level, Katz ADL score, Lawton IADL score, MNA-SF score, and frailty were reevaluated using multivariate regression analysis. Frailty, Katz ADL scores, and Lawton IADL scores were added individually and consecutively in three different regression models (Table V) because of multicollinearity. Old age was independently associated with delirium in all models (OR: 1.095, 95\% CI: [0.011-0.827]; $\mathrm{p}=0.033$ in Model 3). Low Katz ADL score (OR: 0.770, 95\% CI: [0.602-0.985]; $\mathrm{p}=0.037$ ) and low MNA-SF score (OR: 0.583, 95\% CI: [0.357-0.951]; $\mathrm{p}=0.031$ ) were also independently associated with delirium (Table V).

Table IV. Variables associated with delirium according to univariate logistic regression analysis

\begin{tabular}{lccc} 
Variables & OR & CI 95\% & p value \\
\hline Age & 1.093 & $1.016-1.175$ & 0.17 \\
Age $>70$ years & 0.261 & $0.704-0.919$ & 0.036 \\
Number of drugs & 1.179 & $1.013-1.372$ & 0.033 \\
IUC & 0.210 & $0.065-0.679$ & 0.009 \\
Albumin & 0.131 & $0.032-0.536$ & 0.005 \\
\hline Katz ADL & 0.679 & $0.565-0.816$ & 0.000 \\
\hline Lawton IADL & 0.661 & $0.504-0.867$ & 0.003 \\
MNA-SF & 0.598 & $0.449-0.797$ & 0.000 \\
\hline
\end{tabular}

OR: odds ratio, CI: confidence interval, IUC: indwelling urinary catheter, $A D L$ : activities of daily living, IADL: instrumental activities of daily living, MNA-SF: mini nutritional assessment short form. 
Table V. Multivariate regression analysis: Association between delirium and increased age, malnutrition and lower functionality in activities of daily living after adjusting for confounders

\begin{tabular}{|c|c|c|c|}
\hline & Model & Model 2 & Model 3 \\
\hline & \multicolumn{3}{|c|}{ Odds ratios [95\% confidence interval] } \\
\hline Age $\geq 70$ years & $0.124[0.017-0.904]^{\text {la }}$ & $0.087[0.10-0.790]^{2 a}$ & $1.095[0.011-0.827]^{3 a}$ \\
\hline Number of drugs & $1.202[0.950-1.520]$ & $1.146[0.907-1.448]$ & $1.173[0.927-1.483]$ \\
\hline IUC & $0.452[0.091-2.238]$ & $0.578[0.100-3.339]$ & $0.550[0.098-3.073]$ \\
\hline Albumin & $0.528[0.077-3.624]$ & 0.791 [0.094-6.657] & $0.689[0.081-5.854]$ \\
\hline MNA-SF & $0.583[0.357-0.951]^{1 \mathrm{~b}}$ & $0.560[0.331-0.948]^{2 b}$ & $0.560[0.328-0.957]^{3 b}$ \\
\hline Frailty & $0.000[-]$ & & \\
\hline Katz ADL & & $0.770[0.602-0.985]^{2 c}$ & \\
\hline Lawton IADL & & & $0.776[0.588-1.024]$ \\
\hline
\end{tabular}

OR odds ratio, CI confidence interval, IUC indwelling urinary catheter, ADL activities of daily living, IADL instrumental activities of daily living, MNA-SF mini nutritional assessment short form. 1ap $=0.039 ; 1 b p=0.031,2 a p=0.030,2 b p=0.031,2 c p=0.037,3 a p=0.033,3 b p=0.033$

Patients were evaluated in terms of total hospital stay and mortality one month after the index day. A total of $38.9 \%(\mathrm{n}=$ 7) of the patients with delirium and $13.6 \%(n=6)$ of the patients without delirium died within one month (in our hospital or another center) $(\mathrm{p}=0.04)$ (Table VI). According to the results of the survival analysis performed using the Kaplan - Meier method, the mean survival time in patients with delirium was $53 \pm 9.65$ (34.09-71.91) (median survival time (days) + SE [95\% $\mathrm{CI}]$ ). For patients without delirium, the mean survival time was $56+10.44$ (35.54-76.46). There was no statistically significant difference $(\mathrm{p}=0.598)$ (Table VII). The median hospitalization time was longer for patients with delirium (29 (range, 13-80) vs. 18 (range, 5-71) days; $\mathrm{p}=0.029$ ).

Table VI. Mortality of patients with delirium

\begin{tabular}{lccc} 
& $\begin{array}{c}\text { Delirium } \\
\mathrm{n}=18,29 \%\end{array}$ & $\begin{array}{c}\text { No delirium } \\
\mathrm{n}=44,71 \%\end{array}$ & p value \\
\hline Mortality within a month, $\mathrm{n}(\%)$ & $7(38.9)$ & $\mathbf{6 ( 1 3 . 6 )}$ & $\mathbf{0 . 0 4 0}$ \\
\hline
\end{tabular}

$\wedge$ Fischer's exact test

Table VII. Kaplan Meier survival analysis

\begin{tabular}{lccc|} 
& Median (days) & SE & CI 95\% \\
\hline Patients with delirium $(\mathrm{n}=18)$ & 53 & 9.65 & $34.09-71.91$ \\
\hline Patients without delirium $(\mathrm{n}=44)$ & 56 & 10.44 & $35.54-76.46$ \\
\hline
\end{tabular}

SE: standard error, CI: confidence interval

\section{DISCUSSION}

In this study, we investigated delirium risk factors in hospitalized patients aged 60 years and over. All patients included in the study were either pre-frail or frail, in line with other studies in geriatric inpatients [17]. Our delirium rate was 29\%, which was also consistent with a previous study [18]. All patients with delirium were frail. Similar results have been reported in the literature, indicating a strong association between frailty - in comparison with being pre-frail - and delirium [1921]. Frailty is a geriatric syndrome caused by a decrease in the individual's response to stress factors. It is associated with increased falls, long-term hospitalization, sarcopenia, delirium, and even mortality [22]. Frailty occurs in one-third of older adults when they are hospitalized. Even if the patient is treated successfully, frailty persists and increases mortality. Delirium and frailty are common geriatric syndromes with similar risks and outcomes in the hospitalized older adults [20, 23]. Delirium may delay physical and cognitive healing, both of which lead to frailty [19]. At the cellular level, microglial cells in the brain, which respond to inflammation and injury, become hyper-sensitive to stimuli in frail individuals, causing neuronal damage [24]. Hyper-responsive microglia also play a significant role in the pathophysiology of delirium, linking the two geriatric syndromes together [25].

In line with the literature, we found dementia and older age (over 70 years) to be strongly associated with delirium [26, 27]. There was a statistically significant difference between patients with and without delirium concerning their ages. Older age remained as an independent risk factor for delirium in multivariate logistic regression analysis. This relationship can be explained by age-related microvascular changes in the brain, which render the individual more susceptible to neurocognitive disorders. The pre-existing neurodegeneration of dementia also creates a predisposition to delirium. In a meta-analysis examining the association between delirium and dementia, delirium superimposed on dementia was observed at a rate of $22-89 \%$ [28]. In our study, delirium developed in all five patients who had dementia. Common mechanisms underlying the pathogenesis of delirium and cognitive impairment, which include inflammation, atherosclerosis, and malnutrition, may account for this strong association [29].

Physical restraints, another risk factor for delirium, have been reported to increase delirium risk up to 4.5 times [2, 30]. In our study, delirium was detected in all physically restrained patients. It is of note that $50 \%$ of these patients were on antipsychotic medication. Therefore, a reverse causality between delirium and physical restraints is also possible.

A cohort study was conducted by Inouye et al. to develop a model that could predict delirium development and they found five independent precipitating factors: physical restraints, the 
addition of more than three medications, urinary catheterization, malnutrition, and any iatrogenic events [31]. According to this study, the presence of a urinary catheter was found to increase delirium risk by 2.4 -fold [31]. Bo et al's study, which consisted of 1867 hospitalized patients and 1464 nursing home residents, showed that urinary catheterization was independently associated with delirium in hospitalized patients [26]. In accordance with previous studies, we found that the presence of a urinary catheter was associated with delirium. The high rate of urinary catheter use in our clinic may be explained by the multiple comorbidities of hospitalized patients. To prevent delirium, the Hospital Elder Life Program (HELP) and National Institute for Health and Care Excellence (NICE) guidelines recommend avoiding unnecessary urinary catheter use [32, 33]. Urinary catheterization was not an independent risk factor in our study, which may be due to our small sample size.

Studies have shown that polypharmacy is a risk factor for delirium in geriatrics [24]. In the study conducted by Hein et al., the average number of drugs used by 410 older patients in an acute geriatric ward admission was 6.21 , where $69 \%$ of patients with polypharmacy developed delirium. As a result, polypharmacy was found to be an independent risk factor for delirium in older patients, regardless of the type of medication [34]. Similar to previous studies, we found that patients with delirium were on a higher number of medications [2]. However, the number of drugs was not independently associated with delirium. Contrary to previous studies, we had a higher polypharmacy rate, possibly because the study was conducted in a tertiary healthcare center.

Some drug groups including anticholinergics, dopaminergics, sedative-hypnotics, narcotics, $\mathrm{H} 2$ receptor antagonists, antihistamines, fluoroquinolones and analgesics are considered more deliriogenic than others. Among these medications, antipsychotics are the most controversial. Despite their use in delirium treatment, some studies suggest that antipsychotics are deliriogenic [35]. In our study, all patients receiving antipsychotics had delirium. Enteral and parenteral nutrition products were also used more commonly in patients with delirium. This finding is in accordance with studies showing that malnutrition is a risk factor for delirium because nutritional products are mostly used in poor nutritional status [36]. HELP has similarly demonstrated that avoiding malnutrition is one of the effective non - pharmacologic interventions for delirium prevention $[37,38]$. As mentioned before, malnutrition is involved in the pathogenesis of delirium. Mazzola et al., revealed that malnutrition risk and malnutrition were independent determinants of postoperative delirium in patients undergoing hip surgery [39]. MNA-SF is a test with $97.9 \%$ sensitivity, $100 \%$ specificity, and $98.7 \%$ diagnostic accuracy $[11,40]$. In our study, malnutrition was detected in $88.9 \%$ and malnutrition risk in $11.1 \%$ of patients using MNA-SF. Multivariate regression analysis adjusted for old age and functional impairment showed that delirium was more likely in patients with low MNA-SF scores. Serum albumin, a laboratory indicator of nutritional status, was also lower in patients with delirium.
The Katz ADL and Lawton IADL are indices used to assess functional status in older adults $[12,24]$. In a cohort study of 374 patients aged over 65 years, Carrasco et al., found that delirium was independently associated with low functional status as determined with the Barthel index [41]. In a meta-analysis performed by Watt et al. after elective surgery, impairment in $\mathrm{ADL}$, and impairment in IADL were predictors of delirium [42]. In our study, multivariate regression analysis adjusted for low MNA-SF score, older age, and polypharmacy showed that delirium was more likely in patients with low ADL scores. On the other hand, delirium itself may also cause ADL and IADL impairment.

It is also of note that $72 \%$ of the patients with delirium had the hypoactive subtype. Hypoactive delirium is more prevalent and is associated with a greater risk for mortality compared to the hyperactive subtype [43]. This may be due to the fact that unlike the hyperactive subtype, hypoactive delirium tends to go unnoticed. It may also be that patients who are more frail at baseline are unable to present with signs of psychomotor agitation. Thus, clinicians who care for older adults should be on the lookout for signs of hypoactive delirium, which include sleepy appearance, slow response or lack of communication.

Previous studies reported an association between delirium and mortality. In Adamis et al.s cohort study of 164 patients, delirium was not related to in-hospital and 6-month mortality [18]. Likewise, in a review by Hamillton et al. on postoperative delirium, delirium was not associated with mortality in studies that controlled for confounders [44]. In contrast with this information, some studies show that delirium is effective in predicting 3 or even 12-month mortality [20, 45-48]. We evaluated mortality 30 days after the index date using an electronic database. Although mortality rates were found to be significantly higher in patients with delirium, the same significance was not reached in Kruskal-Wallis survival analysis. The results of our study are compatible with studies that found no relationship between delirium and mortality, though our short follow-up period may also be accountable.

Additional healthcare costs are another detrimental effect of delirium [49]. Most health expenses related to delirium are due to prolonged hospitalization of patients. In Siddiqi et al.s review of 42 cohort studies, delirium was associated with longer hospital stay [8], which is in keeping with our results.

Our study has some limitations. First, the sample group was relatively small and the study was conducted in a single center. Thus, our findings cannot be generalized to the entire population. Second, patients were evaluated at a single point in time, which may have resulted in underdiagnosis of delirium. To overcome this obstacle, a delirium chart can be created for the entire healthcare team, including night-shift healthcare workers. Third, our study group consisted only of pre-frail and frail patients, as expected of a tertiary healthcare institution. Therefore, the difference in delirium prevalence between nonfrail and frail patients could not be accurately investigated. Finally, Cox regression analysis could not be performed because mortality was evaluated one month after the index date. Multicenter studies with longer follow - up periods 
may reveal more accurate associations between delirium and mortality.

Our study also has several strengths. To the best of our knowledge, this is the first point prevalence study of delirium in Turkey using CAM. World Delirium Awareness Day was chosen as the index date of our study to draw attention to delirium. Moreover, geriatric syndromes including frailty, malnutrition, polypharmacy, and functional impairment were assessed simultaneously.

With the advent of the COVID-19 pandemic, where older adults have elevated rates of hospitalization, delirium awareness has become more important than ever. Our study revealed that delirium is independently associated with older age, malnutrition, and functional impairment in hospitalized older patients. We recommend that delirium prevention strategies should be developed to minimize modifiable risk factors. Highrisk patients should be screened for delirium at regular intervals and information pamphlets should be given to caregivers, as well as healthcare professionals.

\section{Compliance with the Ethical Standards}

Ethical Approval: The study was approved by Clinical Research Ethics Committee of Marmara University, School of Medicine (Protocol code: 09.2019.273). Written informed consent was obtained from all participants. For patients who were unable to give informed consent, written informed consent was provided by a proxy on behalf of the patient.

Financial Support: The authors have no relevant financial information to close.

Conflict of Interest: The authors have no potential conflicts of interest to disclose

Author Contributions: RGU: Contributed to study design, acquisition of data, analysis and interpretation of data, and preparation of article, BC: Contributed to study design and preparation of article, BI: Contributed to study concept and design, analysis and interpretation of data, and preparation of article, AT: Contributed to study concept and design, analysis and interpretation of data, and preparation of article. All authors read and approved the final article.

\section{REFERENCES}

[1] Savikko N, Pitkala KH, Strandberg TE, Tilvis RS, Laurila JV. Diagnostic agreement on delirium between the Diagnostic and Statistical Manual of Mental Disorders, Fourth Edition; International Classification of Diseases, Tenth Revision; confusion assessment method [CAM]; and the five-item CAM in older adults with dementia. J Am Geriatr Soc 2013;61:6624. doi: 10.1111/jgs.12184

[2] Inouye SK, Westendorp RG, Saczynski JS. Delirium in elderly people. Lancet 2014;383[9920]:911-22. doi: 10.1016/S01406736(13)60688-1

[3] Dani M, Owen LH, Jackson TA, Rockwood K, Sampson EL, Davis D. Delirium, frailty, and mortality: Interactions in a prospective study of hospitalized older people. J Gerontol A Biol Sci Med Sci 2018;73:415-8. doi:10.1093/gerona/glx214

[4] Witlox J, Eurelings LS, de Jonghe JF, Kalisvaart KJ, Eikelenboom P, van Gool WA. Delirium in elderly patients and the risk of postdischarge mortality, institutionalization, and dementia: a meta-analysis. JAMA 2010;304:443-51. doi: 10.1001/jama.2010.1013

[5] Bellelli G, Mazzola P, Morandi A, et al. Duration of postoperative delirium is an independent predictor of 6-month mortality in older adults after hip fracture. J Am Geriatr Soc 2014;62:1335-40. doi: 10.1111/jgs.12885.

[6] Leslie DL, Marcantonio ER, Zhang Y, Leo-Summers L, Inouye SK. One-year health care costs associated with delirium in the elderly population. Arch Intern Med 2008;168:27-32. doi: 10.1001/archinternmed.2007.4

[7] Holt R, Young J, Heseltine D. Effectiveness of a multicomponent intervention to reduce delirium incidence in elderly care wards. Age Ageing 2013;42:721-7. doi: 10.1093/ ageing/aft120

[8] Siddiqi N, House AO, Holmes JD. Occurrence and outcome of delirium in medical in - patients: a systematic literature review. Age Ageing 2006;35:350-64. doi: 10.1093/ageing/ afl005

[9] de la Cruz M, Fan J, Yennu S, et al. The frequency of missed delirium in patients referred to palliative care in a comprehensive cancer center. Support Care Cancer 2015;23:2427-33. doi: 10.1007/s00520.015.2610-3

[10] Guigoz Y, Vellas BJ. Malnutrition in the elderly: the Mini Nutritional Assessment [MNA]. Ther Umsch Rev Ther 1997;54:345-50.

[11] Rubenstein LZ, Harker JO, Salva A, Guigoz Y, Vellas B. Screening for undernutrition in geriatric practice: developing the short-form mini-nutritional assessment [MNA-SF]. J Gerontol A Biol Sci Med Sci 2001;56:M366-72. doi: 10.1093/ gerona/56.6.m 366

[12] Katz S, Ford AB, Moskowitz RW, Jackson BA, Jaffe MW. Studies of illness in the aged. The index of Adl: A standardized measure of biological and psychosocial function. JAMA 1963;185:914-9. doi: 10.1001/jama.1963.030.60120024016

[13] Lawton MP, Brody EM. Assessment of older people: selfmaintaining and instrumental activities of daily living. Gerontologist 1969;9:179-86.

[14] Masnoon N, Shakib S, Kalisch-Ellett L, Caughey GE. What is polypharmacy? A systematic review of definitions. BMC Geriatr 2017;17:230. doi: 10.1186/s12877-017- 0621-2

[15] Morley JE, Malmstrom TK, Miller DK. A simple frailty questionnaire [FRAIL] predicts outcomes in middle aged African Americans. J Nutr Health Aging 2012;16:601-8. doi: 10.1007/s12603.012.0084-2

[16] Inouye SK, van Dyck CH, Alessi CA, Balkin S, Siegal AP, Horwitz RI. Clarifying confusion: the confusion assessment method. A new method for detection of delirium. Ann Intern Med 1990;113:941-8. doi: 10.7326/0003-4819-113-12-941

[17] Joosten E, Demuynck M, Detroyer E, Milisen K. Prevalence of frailty and its ability to predict in hospital delirium, falls, 
and 6-month mortality in hospitalized older patients. BMC Geriatr 2014;14:1. doi: 10.1186/1471-2318-14-1

[18] Adamis D, Treloar A, Darwiche FZ, Gregson N, Macdonald AJ, Martin FC. Associations of delirium with in-hospital and in 6-months mortality in elderly medical inpatients. Age Ageing 2007;36:644-9. doi: 10.1093/ageing/afm094

[19] Verloo H, Goulet C, Morin D, von Gunten A. Association between frailty and delirium in older adult patients discharged from hospital. Clin Interv Aging 2016;11:55-63. doi: 10.2147/ CIA.S100576

[20] Eeles EM, White SV, O’Mahony SM, Bayer AJ, Hubbard RE. The impact of frailty and delirium on mortality in older inpatients. Age Ageing 2012;41:412-6. doi: 10.1093/ageing/ afs021

[21] Hubbard RE, Peel NM, Samanta M, Gray LC, Mitnitski A, Rockwood K. Frailty status at admission to hospital predicts multiple adverse outcomes. Age Ageing 2017;46:801-6. doi: 10.1093/ageing/afx081

[22] Cesari M, Calvani R, Marzetti E. Frailty in Older Persons. Clin Geriatr Med 2017;33:293-303. doi: 10.1016/j.cger.2017.02.002

[23] Leung JM, Tsai TL, Sands LP. Brief report: preoperative frailty in older surgical patients is associated with early postoperative delirium. Anesth Analg 2011;112:1199-201. doi: 10.1213/ ANE.0b013e31820c7c06

[24] Clegg A, Young J, Iliffe S, Rikkert MO, Rockwood K. Frailty in elderly people. Lancet 2013;381:752-62. doi: 10.1016/S01406736(12)62167-9

[25] van Gool WA, van de Beek D, Eikelenboom P. Systemic infection and delirium: when cytokines and acetylcholine collide. Lancet 2010;375:773-5. doi: 10.1016/S01406736(09)61158-2

[26] Bo M, Porrino P, Di Santo SG, Mazzone A, Cherubini A, et al. The association of indwelling urinary catheter with delirium in hospitalized patients and nursing home residents: an explorative analysis from the "Delirium Day 2015". Aging Clin Exp Res 2019;31:411-20. doi: 10.3390/jpm11060445

[27] O’Regan NA, Fitzgerald J, Adamis D, Molloy DW, Meagher D, Timmons S. Predictors of Delirium Development in Older Medical Inpatients: Readily Identifiable Factors at Admission. J Alzheimers Dis 2018;64:775-85. doi: 10.3233/jad-180178

[28] Fick DM, Agostini JV, Inouye SK. Delirium superimposed on dementia: a systematic review. J Am Geriatr Soc 2002;50:172332. doi:10.1046/j.1532-5415.2002.50468.x

[29] Cunningham C. Systemic inflammation and delirium: important co-factors in the progression of dementia. Biochem Soc Trans 2011;39:945-53. doi:10.1042/BST0390945

[30] Lach HW, Leach KM, Butcher HK. Evidence-Based Practice Guideline: Changing the Practice of Physical Restraint Use in Acute Care. J Gerontol Nurs 2016;42:17-26. doi:10.3928/00989.134.20160113-04

[31] Inouye SK, Charpentier PA. Precipitating factors for delirium in hospitalized elderly persons. Predictive model and interrelationship with baseline vulnerability. JAMA 1996;275:852-7. PMID: 8596223.
[32] Young J, Murthy L, Westby M, Akunne A, O’Mahony R. Diagnosis, prevention, and management of delirium: summary of NICE guidance. BMJ 2010;341:c3704. PMID: 8596223.

[33] Yue J, Tabloski P, Dowal SL, Puelle MR, Nandan R, Inouye SK. NICE to HELP: operationalizing National Institute for Health and Clinical Excellence guidelines to improve clinical practice. J Am Geriatr Soc 2014;62:754-61. doi:10.1111/jgs.12768

[34] Hein C, Forgues A, Piau A, Sommet A, Vellas B, Nourhashemi F. Impact of polypharmacy on occurrence of delirium in elderly emergency patients. J Am Med Dir Assoc 2014;15:850 e11-5. doi:10.1016/j.jamda.2014.08.012

[35] Trzepacz PT. Update on the neuropathogenesis of delirium. Dement Geriatr Cogn Disord 1999;10:330-4. doi: 10.1159/000017164

[36] van Eijsden WA, Raats JW, Mulder PG, van der Laan L. New aspects of delirium in elderly patients with critical limb ischemia. Clin Interv Aging 2015;10:1537-46. doi: 10.2147/ CIA.S87383

[37] Inouye SK, Baker DI, Fugal P, Bradley EH. Dissemination of the hospital elder life program: implementation, adaptation, and successes. J Am Geriatr Soc 2006;54:1492-9. doi:10.1111/ j.1532-5415.2006.00869.x

[38] Leslie DL, Zhang Y, Bogardus ST, Holford TR, Leo-Summers LS, Inouye SK. Consequences of preventing delirium in hospitalized older adults on nursing home costs. J Am Geriatr Soc 2005;53:405-9. doi: 10.1111/j.1532-5415.2005.53156.x

[39] Mazzola P, Ward L, Zazzetta S, et al. Association Between Preoperative Malnutrition and Postoperative Delirium After Hip Fracture Surgery in Older Adults. J Am Geriatr Soc 2017;65:1222-8. doi: 10.1111/jgs.14764

[40] Montejano Lozoya R, Martinez-Alzamora N, Clemente Marin G, Guirao-Goris SJA, Ferrer-Diego RM. Predictive ability of the Mini Nutritional Assessment Short Form [MNA-SF] in a free-living elderly population: a cross-sectional study. PeerJ 2017;5:e3345. doi: 10.7717/peerj.3345

[41] Carrasco MP, Villarroel L, Andrade M, Calderon J, Gonzalez M. Development and validation of a delirium predictive score in older people. Age Ageing 2014;43:346-51. doi: 10.1093/ ageing/aft141

[42] Watt J, Tricco AC, Talbot-Hamon C, et al. Identifying Older Adults at Risk of Delirium Following Elective Surgery: A Systematic Review and Meta-Analysis. J Gen Intern Med 2018;33:500-9. doi: 10.1007/s11606.017.4204-x

[43] Kim SY, Kim SW, Kim JM, et al. Differential associations between delirium and mortality according to delirium subtype and age: A Prospective Cohort Study. Psychosom Med 2015; 77:903-910. doi: 10.1097/PSY.000.000.0000000239

[44] Hamilton GM, Wheeler K, Di Michele J, Lalu MM, McIsaac DI. A Systematic Review and Meta-analysis Examining the Impact of Incident Postoperative Delirium on Mortality. Anesthesiology 2017;127:78-88. doi: 10.1097/ ALN.000.000.0000001660

[45] Dharmarajan K, Swami S, Gou RY, Jones RN, Inouye SK. Pathway from Delirium to Death: Potential In-Hospital 
Mediators of Excess Mortality. J Am Geriatr Soc 2017;65:102633. doi: 10.1111/jgs.14743.

[46] Pendlebury ST, Lovett NG, Smith SC, et al. Observational, longitudinal study of delirium in consecutive unselected acute medical admissions: age-specific rates and associated factors, mortality and re-admission. BMJ Open 2015;5:e007808. doi: 10.1136/bmjopen- 2015-007808.

[47] Morandi A, Di Santo SG, Zambon A, et al. Delirium, Dementia, and In-Hospital Mortality: The Results From the
Italian Delirium Day 2016, A National Multicenter Study. J Gerontol A Biol Sci Med Sci 2019;74:910-6. doi: 10.1093/ gerona/gly154.

[48] McCusker J, Cole M, Abrahamowicz M, Primeau F, Belzile E. Delirium predicts 12 - month mortality. Arch Intern Med 2002;162:457-63. doi:10.1001/archinte.162.4.457

[49] Oh ES, Fong TG, Hshieh TT, Inouye SK. Delirium in Older Persons: Advances in Diagnosis and Treatment. JAMA 2017;318:1161-74. doi: 10.1001/jama.2017.12067.

Supplementary Table I. Medical characteristics of patients ( $n=62)$

\section{Variables}

BMI, median (min-max)

Diagnosis, $\mathbf{n}(\%)$

Malignancy research

Respiratory diseases

Gastrointestinal system diseases

Acute kidney injury

Infectious diseases

Any prior tumor

Others

Comorbidities, $\mathbf{n}(\%)$

Follow up after intensive care stay

Dementia

Malignancies

COPD

Pulmonary embolism

Diabetes

Chronic kidney disease

Hemodialysis

Coronary artery disease

Heart failure

Atrial fibrillation

Hypertension

Hypothyroidism

Others

BMI: body mass index; COPD: chronic obstructive pulmonary disease

\section{Values}

$24.8(15.6-39.1)$

$7(11.3)$

$21(33.9)$

$5(8.1)$

$10(16.1)$

$5(8.1)$

$9(14.5)$

$5(8.1)$

$13(21)$

$5(8.1)$

$29(46.8)$

$19(30.6)$

$11(17.7)$

$21(35.5)$

$13(21)$

$6(9.7)$

$17(27.4)$

$11(17.7)$

$11(17.7)$

$31(50)$

$6(9.7)$

$8(12.9)$

Supplementary Table II. Laboratory findings of patients $(n=62)$

\section{Variable}

Creatinine, median (mg/dl) (min-max)

GFR, median ( $\mathrm{ml} / \mathrm{min})$ (Cockroft) (min-max)

Hemoglobin, median (g/dl) (min-max)

CRP, median (mg/L) (min-max)

Sodium, median $(\mathrm{mEq} / \mathrm{L})(\min -\mathrm{max})$

Albumin, median (g/L) (min-max)

GFR: glomerular filtration rate, CRP: C-reactive serum protein 